\title{
Conditional Stability and Asymptotic Behavior of Solutions of Weakly Delayed Linear Discrete Systems in $\mathbb{R}^{2}$
}

\author{
Josef Diblík, ${ }^{1}$ Hana Halfarová, ${ }^{1}$ and Jan Šafařík ${ }^{1,2}$ \\ ${ }^{1}$ Department of Mathematics and Descriptive Geometry, Faculty of Civil Engineering, Brno University of Technology, \\ Brno, Czech Republic \\ ${ }^{2}$ Department of Mathematics, Faculty of Electrical Engineering and Communication, Brno University of Technology, \\ Brno, Czech Republic \\ Correspondence should be addressed to Josef Diblík; diblik.j@fce.vutbr.cz
}

Received 27 February 2017; Accepted 28 March 2017; Published 11 June 2017

Academic Editor: Garyfalos Papashinopoulos

Copyright ( 92017 Josef Diblík et al. This is an open access article distributed under the Creative Commons Attribution License, which permits unrestricted use, distribution, and reproduction in any medium, provided the original work is properly cited.

Two-dimensional linear discrete systems $x(k+1)=A x(k)+\sum_{l=1}^{n} B_{l} x_{l}\left(k-m_{l}\right), k \geq 0$, are analyzed, where $m_{1}, m_{2}, \ldots, m_{n}$ are constant integer delays, $0<m_{1}<m_{2}<\cdots<m_{n}, A, B_{1}, \ldots, B_{n}$ are constant $2 \times 2$ matrices, $A=\left(a_{i j}\right), B_{l}=\left(b_{i j}^{l}\right), i, j=1,2, l=1,2, \ldots, n$, and $x:\left\{-m_{n},-m_{n}+1, \ldots\right\} \rightarrow \mathbb{R}^{2}$. Under the assumption that the system is weakly delayed, the asymptotic behavior of its solutions is studied and asymptotic formulas are derived.

\section{Introduction and Preliminaries}

Throughout the paper, we use the notations: $\Theta$ is zero $2 \times 2$ matrix, $I$ is the unit $2 \times 2$ matrix, $\theta$ is zero $2 \times 1$ vector, and $\mathbb{Z}_{s}^{q}=\{s, s+1, \ldots, q\}$, where $s$ and $q$ are integers, $s \leq q$. Similarly, a set $\mathbb{Z}_{s}^{\infty}$ is defined. delays

In the paper, we investigate discrete planar systems with

$$
x(k+1)=A x(k)+\sum_{l=1}^{n} B_{l} x_{l}\left(k-m_{l}\right),
$$

where $m_{1}, m_{2}, \ldots, m_{n}$ are integers such that $0<m_{1}<m_{2}<$ $\cdots<m_{n}, k \in \mathbb{Z}_{0}^{\infty}, A, B_{1}, \ldots, B_{n}$ are constant $2 \times 2$ matrices, $A=\left(a_{i j}\right), B_{l}=\left(b_{i j}^{l}\right), i, j=1,2, l=1,2, \ldots, n$,

$$
B_{l} \neq \Theta, \quad l=1,2, \ldots, n,
$$

and $x: \mathbb{Z}_{-m_{n}}^{\infty} \rightarrow \mathbb{R}^{2}$. An initial problem to (1) is

$$
x(k)=\varphi(k),
$$

where $k=-m_{n},-m_{n}+1, \ldots, 0$ and $\varphi: \mathbb{Z}_{-m_{n}}^{0} \rightarrow \mathbb{R}^{2}$. Obviously, the initial problem (1), (3) has a unique solution on $\mathbb{Z}_{-m_{n}}^{\infty}$.
1.1. Weakly Delayed Systems. It is well-known that the characteristic equation to (1) is

$$
D(\lambda):=\operatorname{det}\left(A+\sum_{l=1}^{n} \lambda^{-m_{l}} B_{l}-\lambda I\right)=0,
$$

where $\lambda \in \mathbb{C}$, and the characteristic equation to a system without delays

$$
x(k+1)=A x(k)
$$

is

$$
\operatorname{det}(A-\lambda I)=0 .
$$

The following definition and lemma are taken from [1].

Definition 1. System (1) is called weakly delayed if equations (4) and (6) are equal, that is, if

$$
D(\lambda)=\operatorname{det}(A-\lambda I), \quad \forall \lambda \in \mathbb{C} \backslash\{0\} .
$$

Lemma 2. If system (1) is a weakly delayed system, then its arbitrary linear nonsingular transformation $x(k)=\delta y(k)$ with $2 \times 2$ matrix $\mathcal{S}$ is again a weakly delayed system. 
In [1], the following necessary and sufficient conditions determining weakly delayed systems are also derived.

Theorem 3. System (1) is a weakly delayed system if and only if

$$
\begin{array}{r}
b_{11}^{l}+b_{22}^{l}=0, \\
\left|\begin{array}{ll}
b_{11}^{l} & b_{12}^{l} \\
b_{21}^{l} & b_{22}^{l}
\end{array}\right|=0, \\
\left|\begin{array}{ll}
a_{11} & a_{12} \\
b_{21}^{l} & b_{22}^{l}
\end{array}\right|+\left|\begin{array}{ll}
b_{11}^{l} & b_{12}^{l} \\
a_{21} & a_{22}
\end{array}\right|=0, \\
\left|\begin{array}{ll}
b_{11}^{l} & b_{12}^{l} \\
b_{21}^{v} & b_{22}^{v}
\end{array}\right|+\left|\begin{array}{ll}
b_{11}^{v} & b_{12}^{v} \\
b_{21}^{l} & b_{22}^{l}
\end{array}\right|
\end{array}
$$

where $l, v=1,2, \ldots, n$ and $v>l$.

1.2. Problem under Consideration. In the paper (in Section 2), we are concerned with conditional stability of (1) with the results formulated in Theorems 11-18. In Section 3, asymptotic formulas describing the behavior of solutions (for $k \rightarrow$ $\infty)$ of nontrivial solutions of (1) are derived with the results formulated in Theorems 19-24.

To prove such results, we use explicit analytic formulas on the representation of solutions, derived by the first two authors in [1] and, for the reader's convenience, we recall them in the following part.

\subsection{Representation of Solutions}

1.3.1. Jordan Forms. It is known that, for every matrix $A$, there exists a nonsingular matrix $S$ transforming it to the corresponding Jordan matrix form $\Lambda=S^{-1} A S$. In [[1], Theorem 6], formulas (8)-(11) are analyzed and it is concluded that system (1) can be a weakly delayed system only if matrix $A$ has one of the following three Jordan matrix forms (the case of the roots of (6) being complex conjugate is not compatible with (2) and (8)-(11)):

$$
\Lambda_{1}=\left(\begin{array}{cc}
\lambda_{1} & 0 \\
0 & \lambda_{2}
\end{array}\right)
$$

if (6) has two real distinct roots $\lambda_{1}, \lambda_{2}$, and either

$$
\Lambda_{2}=\left(\begin{array}{ll}
\lambda & 0 \\
0 & \lambda
\end{array}\right)
$$

or

$$
\Lambda_{3}=\left(\begin{array}{ll}
\lambda & 1 \\
0 & \lambda
\end{array}\right)
$$

in the case of one double real root $\lambda_{1,2}=\lambda$. Transforming (1) by $y(k)=S^{-1} x(k)$, we get

$$
y(k+1)=\Lambda y(k)+\sum_{l=1}^{n} B_{l}^{*} y\left(k-m_{l}\right), \quad k \in \mathbb{Z}_{0}^{\infty},
$$

where $B_{l}^{*}=S^{-1} B_{l} S, B_{l}^{*}=\left(b_{i j}^{* l}\right), l=1, \ldots, n$, and $i, j=1,2$. The transformed initial problem for (15) is

$$
y(k)=\varphi^{*}(k)=\left(\varphi_{1}^{*}(k), \varphi_{2}^{*}(k)\right)^{T}:=S^{-1} \varphi(k),
$$

$$
k \in \mathbb{Z}_{-m_{n}}^{0} \text {. }
$$

Below, we will use functions $\Phi_{i}: \mathbb{Z}_{-m_{n}}^{0} \rightarrow \mathbb{R}^{2}, i=1,2$, defined as

$$
\begin{aligned}
& \Phi_{1}(k):=\left(0, \varphi_{1}^{*}(k)\right)^{T}, \\
& \Phi_{2}(k):=\left(\varphi_{2}^{*}(k), 0\right)^{T},
\end{aligned}
$$

$$
k \in \mathbb{Z}_{-m_{n}}^{0}
$$

and functions $\Phi_{l}^{*}: \mathbb{Z}_{-m_{n}}^{0} \rightarrow \mathbb{R}^{2}, l=1,2, \ldots, n$, defined as

$$
\begin{gathered}
\Phi_{l}^{*}(k)=\left(\Phi_{l 1}^{*}(k), \Phi_{l 2}^{*}(k)\right)^{T} \\
:=\left(b_{11}^{* l}\left[\varphi_{1}^{*}(k)+\frac{b_{12}^{* 1}}{b_{11}^{* 1}} \varphi_{2}^{*}(k)\right],\right. \\
\left.-\frac{\left(b_{11}^{* l}\right)^{2}}{b_{12}^{* l}}\left[\varphi_{1}^{*}(k)+\frac{b_{12}^{* 1}}{b_{11}^{* 1}} \varphi_{2}^{*}(k)\right]\right)^{T},
\end{gathered}
$$

where $k \in \mathbb{Z}_{-m_{n}}^{0}, l=1,2, \ldots, n$.

1.3.2. Explicit Analytic Formulas in Case (12). In this case, $\Lambda=$ $\Lambda_{1}, \Lambda_{1}^{k}=\operatorname{diag}\left(\lambda_{1}^{k}, \lambda_{2}^{k}\right)$. From conditions (8)-(11) for (15) it follows (we refer to [[1], Part 2.1.1.]) that either

$$
\text { (I) } b_{11}^{* l}=b_{22}^{* l}=b_{21}^{* l}=0, b_{12}^{* l} \neq 0, l=1,2, \ldots, n,
$$

or

$$
\text { (II) } b_{11}^{* l}=b_{22}^{* l}=b_{12}^{* l}=0, b_{21}^{* l} \neq 0, l=1,2, \ldots, n .
$$

The proofs of the below results are given in [[1], Theorem 5].

Theorem 4. Let (1) be a weakly delayed system and (6) have two real distinct roots $\lambda_{1}, \lambda_{2}$. In case (I), the solution of the initial problem (1), (3) is $x(k)=S y(k), k \in \mathbb{Z}_{-m_{n}}^{\infty}$, where

$$
\begin{aligned}
& \text { (I } \left.\mathrm{I}_{1}\right) y(k)=\varphi^{*}(k) \text { if } k \in \mathbb{Z}_{-m_{n}}^{0} \text {, } \\
& \left(\mathrm{I}_{2}\right) y(k)=\Lambda_{1}^{k} \varphi^{*}(0)+\sum_{r=0}^{k-1} \lambda_{1}^{k-1-r}\left[\sum_{l=1}^{n} b_{12}^{* l} \Phi_{2}\left(r-m_{l}\right)\right] \text {, if } \\
& k \in \mathbb{Z}_{1}^{m_{1}+1} \text {, } \\
& \left(\mathrm{I}_{3}\right) y(k)=\Lambda_{1}^{k} \varphi^{*}(0)+\sum_{r=0}^{k-1} \lambda_{1}^{k-1-r}\left[\sum_{l=s+1}^{n} b_{12}^{* l} \Phi_{2}(r-\right. \\
& \left.\left.m_{l}\right)\right]+\sum_{l=1}^{s} b_{12}^{* l}\left[\sum_{r=0}^{m_{l}} \lambda_{1}^{k-1-r} \Phi_{2}\left(r-m_{l}\right)+\right. \\
& \left.\Phi_{2}(0) \sum_{r=m_{l}+1}^{k-1} \lambda_{1}^{k-1-r} \lambda_{2}^{r-m_{l}}\right] \text { if } k \in \mathbb{Z}_{m_{s}+2}^{m_{s+1}+1}, s=1,2, \ldots \text {, } \\
& n-1 \text {, } \\
& \left(\mathrm{I}_{4}\right) y(k)=\Lambda_{1}^{k} \varphi^{*}(0)+\sum_{l=1}^{n} b_{12}^{* l}\left[\sum_{r=0}^{m_{l}} \lambda_{1}^{k-1-r} \Phi_{2}\left(r-m_{l}\right)+\right. \\
& \left.\Phi_{2}(0) \sum_{r=m_{l}+1}^{k-1} \lambda_{1}^{k-1-r} \lambda_{2}^{r-m_{l}}\right] \text { if } k \in \mathbb{Z}_{m_{n}+2}^{\infty} \text {. }
\end{aligned}
$$

Theorem 5. Let (1) be a weakly delayed system and (6) have two real distinct roots $\lambda_{1}, \lambda_{2}$. In case (II), the solution of the initial problem (1), (3) is $x(k)=S y(k), k \in \mathbb{Z}_{-m_{n}}^{\infty}$, where 


$$
\begin{aligned}
& \text { (II }) y(k)=\varphi^{*}(k), \text { if } k \in \mathbb{Z}_{-m_{n}}^{0} \text {, } \\
& \left(\mathrm{II}_{2}\right) y(k)=\Lambda_{1}^{k} \varphi^{*}(0)+\sum_{r=0}^{k-1} \lambda_{2}^{k-1-r}\left[\sum_{l=1}^{n} b_{21}^{* l} \Phi_{1}\left(r-m_{l}\right)\right] \text {, if } \\
& k \in \mathbb{Z}_{1}^{m_{1}+1}, \\
& \left(\mathrm{II}_{3}\right) y(k)=\Lambda_{1}^{k} \varphi^{*}(0)+\sum_{r=0}^{k-1} \lambda_{2}^{k-1-r}\left[\sum_{l=s+1}^{n} b_{21}^{* l} \Phi_{1}(r-\right. \\
& \left.\left.m_{l}\right)\right]+\sum_{l=1}^{s} b_{21}^{* l}\left[\sum_{r=0}^{m_{l}} \lambda_{2}^{k-1-r} \Phi_{1}\left(r-m_{l}\right) \quad+\right. \\
& \left.\Phi_{1}(0) \sum_{r=m_{l}+1}^{k-1} \lambda_{1}^{r-m_{l}} \lambda_{2}^{k-1-r}\right] \text { if } k \in \mathbb{Z}_{m_{s}+2}^{m_{s+1}+1}, s=1,2, \ldots \text {, } \\
& n-1 \text {, } \\
& \left(\mathrm{II}_{4}\right) y(k)=\Lambda_{1}^{k} \varphi^{*}(0)+\sum_{l=1}^{n} b_{21}^{* l}\left[\sum_{r=0}^{m_{l}} \lambda_{2}^{k-1-r} \Phi_{1}\left(r-m_{l}\right)+\right. \\
& \left.\Phi_{1}(0) \sum_{r=m_{1}+1}^{k-1} \lambda_{1}^{r-m_{l}} \lambda_{2}^{k-1-r}\right] \text { if } k \in \mathbb{Z}_{m_{n}+2}^{\infty} \text {. }
\end{aligned}
$$

1.3.3. Explicit Analytic Formulas in Case (13). We have $\Lambda=$ $\Lambda_{2}, \Lambda_{2}^{k}=\operatorname{diag}\left(\lambda^{k}, \lambda^{k}\right)$ and, from the necessary and sufficient conditions (8)-(11) for (15), it follows (see [[1], Part 2.1.3.]) that only the following three cases are possible:
(a) $b_{11}^{* l}=b_{22}^{* l}=b_{21}^{* l}=0, b_{12}^{* l} \neq 0, l=1,2, \ldots, n$,
(b) $b_{11}^{* l}=b_{22}^{* l}=b_{12}^{* l}=0, b_{21}^{* l} \neq 0, l=1,2, \ldots, n$,
(c) $b_{12}^{* l} b_{21}^{* l} \neq 0, l=1,2, \ldots, n$.

The results formulated below are proved in [[1], Theorems 7 and 8],

Theorem 6. Let (1) be a weakly delayed system, (6) have a twofold root $\lambda_{1,2}=\lambda$, and the matrix $\Lambda$ have the form (13). In case (a), the solution of the initial problem (1), (3) is $x(k)=$ $S y(k), k \in \mathbb{Z}_{-m_{n}}^{\infty}$, where

$$
\begin{aligned}
& \left(\mathrm{a}_{1}\right) y(k)=\varphi^{*}(k), \text { if } k \in \mathbb{Z}_{-m_{n}}^{0}, \\
& \text { (a } \left.\mathrm{a}_{2}\right) y(k)=\Lambda_{2}^{k} \varphi^{*}(0)+\sum_{r=0}^{k-1} \lambda^{k-1-r}\left[\sum_{l=1}^{n} b_{12}^{* l} \Phi_{2}\left(r-m_{l}\right)\right] \text {, if } \\
& k \in \mathbb{Z}_{1}^{m_{1}+1} \text {, } \\
& \left(\mathrm{a}_{3}\right) y(k)=\Lambda_{2}^{k} \varphi^{*}(0)+\sum_{r=0}^{k-1} \lambda^{k-1-r}\left[\sum_{l=s+1}^{n} b_{12}^{* l} \Phi_{2}(r-\right. \\
& \left.\left.m_{l}\right)\right]+\sum_{l=1}^{s} b_{12}^{* l}\left[\sum_{r=0}^{m_{l}} \lambda^{k-1-r} \Phi_{2}\left(r-m_{l}\right)+(k-1-\right. \\
& \left.\left.m_{l}\right) \lambda^{k-1-m_{l}} \Phi_{2}(0)\right] \text { if } k \in \mathbb{Z}_{m_{\mathrm{s}}+2}^{m_{s+1}+1}, s=1,2, \ldots, n-1, \\
& \text { ( } \left.\mathrm{a}_{4}\right) y(k)=\Lambda_{2}^{k} \varphi^{*}(0)+\sum_{l=1}^{n} b_{12}^{* l}\left[\sum_{r=0}^{m_{l}} \lambda^{k-1-r} \Phi_{2}\left(r-m_{l}\right)+\right. \\
& \left.\left(k-1-m_{l}\right) \lambda^{k-1-m_{l}} \Phi_{2}(0)\right] \text { if } k \in \mathbb{Z}_{m_{n}+2}^{\infty} \text {. }
\end{aligned}
$$

Theorem 7. Let (1) be a weakly delayed system, (6) have a twofold root $\lambda_{1,2}=\lambda$, and the matrix $\Lambda$ have the form (13). In case (b), the solution of the initial problem (1), (3) is $x(k)=$ $S y(k), k \in \mathbb{Z}_{-m_{n}}^{\infty}$, where

$$
\begin{aligned}
\left(\mathrm{b}_{1}\right) & y(k)=\varphi^{*}(k), \text { if } k \in \mathbb{Z}_{-m_{n}}^{0}, \\
\left(\mathrm{~b}_{2}\right) & y(k)=\Lambda_{2}^{k} \varphi^{*}(0)+\sum_{r=0}^{k-1} \lambda^{k-1-r}\left[\sum_{l=1}^{n} b_{21}^{* l} \Phi_{1}\left(r-m_{l}\right)\right], \text { if } \\
& k \in \mathbb{Z}_{1}^{m_{1}+1}, \\
\left(\mathrm{~b}_{3}\right) & y(k)=\Lambda_{2}^{k} \varphi^{*}(0)+\sum_{r=0}^{k-1} \lambda^{k-1-r}\left[\sum_{l=s+1}^{n} b_{21}^{* l} \Phi_{1}(r-\right. \\
& \left.\left.m_{l}\right)\right]+\sum_{l=1}^{s} b_{21}^{* l}\left[\sum_{r=0}^{m_{l}} \lambda^{k-1-r} \Phi_{1}\left(r-m_{l}\right)+(k-1-\right. \\
& \left.\left.m_{l}\right) \lambda^{k-1-m_{l}} \Phi_{1}(0)\right] \text { if } k \in \mathbb{Z}_{m_{s}+2}^{m_{s+1}+1}, s=1,2, \ldots, n-1, \\
\left(\mathrm{~b}_{4}\right) & y(k)=\Lambda_{2}^{k} \varphi^{*}(0)+\sum_{l=1}^{n} b_{21}^{* l}\left[\sum_{r=0}^{m_{l}} \lambda^{k-1-r} \Phi_{1}\left(r-m_{l}\right)+\right. \\
& \left.\left(k-1-m_{l}\right) \lambda^{k-1-m_{l}} \Phi_{1}(0)\right] \text { if } k \in \mathbb{Z}_{m_{n}+2}^{\infty} .
\end{aligned}
$$

Theorem 8. Let system (1) be a weakly delayed system, (6) have two repeated roots $\lambda_{1,2}=\lambda$, and the matrix $\Lambda$ have the form (13). In case (c), the solution of the initial problem (1), (3) is $x(k)=S y(k), k \in \mathbb{Z}_{-m_{n}}^{\infty}$, where

$$
\begin{aligned}
\left(\mathrm{c}_{1}\right) & y(k)=\varphi^{*}(k), \text { if } k \in \mathbb{Z}_{-m_{n}}^{0}, \\
\left(\mathrm{c}_{2}\right) & y(k)=\Lambda_{2}^{k} \varphi^{*}(0)+\sum_{r=0}^{k-1} \lambda^{k-1-r}\left[\sum_{l=1}^{n} \Phi_{l}^{*}\left(r-m_{l}\right)\right], \text { if } \\
& k \in \mathbb{Z}_{1}^{m_{1}+1}, \\
\left(\mathrm{c}_{3}\right) & y(k)=\Lambda_{2}^{k} \varphi^{*}(0)+\sum_{r=0}^{k-1} \lambda^{k-1-r}\left[\sum_{l=s+1}^{n} \Phi_{l}^{*}\left(r-m_{l}\right)\right]+ \\
& \sum_{l=1}^{s}\left[\sum_{r=0}^{m_{l}} \lambda^{k-1-r} \Phi_{l}^{*}\left(r-m_{l}\right)+\left(k-1-m_{l}\right) \lambda^{k-1-m_{l}} \Phi_{l}^{*}(0)\right] \\
& \text { if } k \in \mathbb{Z}_{m_{s}+2}^{m_{s+1}+1}, s=1,2, \ldots, n-1, \\
\left(\mathrm{c}_{4}\right) & y(k)=\Lambda_{2}^{k} \varphi^{*}(0)+\sum_{l=1}^{n}\left[\sum_{r=0}^{m_{l}} \lambda^{k-1-r} \Phi_{l}^{*}\left(r-m_{l}\right)+(k-\right. \\
& \left.\left.1-m_{l}\right) \lambda^{k-1-m_{l}} \Phi_{l}^{*}(0)\right] \text { if } k \in \mathbb{Z}_{m_{n}+2}^{\infty} .
\end{aligned}
$$

1.3.4. Explicit Analytic Formulas in Case (14). We have $\Lambda=$ $\Lambda_{3}$. The necessary and sufficient conditions (8)-(11) for (15) are reduced (we refer to [[1], Part 2.1.6.]) to $b_{11}^{* l}=b_{22}^{* l}=b_{21}^{* l}=$ $0, b_{12}^{* l} \neq 0, l=1,2, \ldots, n$. The following result is proved in [[1], Theorem 9].

Theorem 9. Let (1) be a weakly delayed system, (6) have a double root $\lambda_{1,2}=\lambda$, and the matrix $\Lambda$ have the form (14). Then, the solution of the initial problem (1), (3) is $x(k)=$ $S y(k)=S\left(y_{1}(k), y_{2}(k)\right)^{T}, k \in \mathbb{Z}_{-m_{n}}^{\infty}$, where

$$
\begin{aligned}
& 1\left(\mathrm{y}_{1} \mathrm{y}_{2}\right) y_{1}(k)=\varphi_{1}^{*}(k), \quad y_{2}(k)=\varphi_{2}^{*}(k), \text { if } k \in \mathbb{Z}_{-m_{n}}^{0}, \\
& 2\left(\mathrm{y}_{1}\right) y_{1}(k)=\lambda^{k} \varphi_{1}^{*}(0)+k \lambda^{k-1} \varphi_{2}^{*}(0)+ \\
& \sum_{r=0}^{k-1} \lambda^{k-1-r}\left[\sum_{l=1}^{n} b_{12}^{* l} \varphi_{2}^{*}\left(r-m_{l}\right)\right], \text { if } k \in \mathbb{Z}_{1}^{m_{1}+1}, \\
& 3\left(\mathrm{y}_{1}\right) y_{1}(k)=\lambda^{k} \varphi_{1}^{*}(0)+k \lambda^{k-1} \varphi_{2}^{*}(0)+ \\
& \sum_{r=0}^{k-1} \lambda^{k-1-r}\left[\sum_{l=s+1}^{n} b_{12}^{* l} \varphi_{2}^{*}\left(r \quad-m_{l}\right)\right] \quad+ \\
& \sum_{l=1}^{s} b_{12}^{* l}\left[\sum_{r=0}^{m_{l}} \lambda^{k-1-r} \varphi_{2}^{*}\left(r-m_{l}\right)+(k-1-\right. \\
& \left.\left.m_{l}\right) \lambda^{k-1-m_{l}} \varphi_{2}^{*}(0)\right] \text { if } k \in \mathbb{Z}_{m_{s}+2}^{m_{s+1}+1}, s=1,2, \ldots, n-1, \\
& \begin{aligned}
4\left(\mathrm{y}_{1}\right) & y_{1}(k)=\lambda^{k} \varphi_{1}^{*}(0)+k \lambda^{k-1} \varphi_{2}^{*}(0)+ \\
& \sum_{l=1}^{n} b_{12}^{* l}\left[\sum_{r=0}^{m_{l}} \lambda^{k-1-r} \varphi_{2}^{*}\left(r-m_{l}\right)+(k-1-\right. \\
& \left.\left.m_{l}\right) \lambda^{k-1-m_{l}} \varphi_{2}^{*}(0)\right] \text { if } k \in \mathbb{Z}_{m_{n}+2}^{\infty},
\end{aligned} \\
& 5\left(\mathrm{y}_{2}\right) y_{2}(k)=\lambda^{k} \varphi_{2}^{*}(0) \text { if } k \in \mathbb{Z}_{1}^{\infty} \text {. }
\end{aligned}
$$

\section{Conditional Stability}

In [[1], Theorem 10], it is explained that the space of solutions of a weakly delayed system (1), depending initially on $2\left(m_{n}+\right.$ 1) parameters (i.e., on the initial data (3)) is reduced (as $k \geq$ $m_{n}+2$ ) to a space of solutions depending either on $m_{n}+1$ or even only on 2 parameters. This is also visible from an analysis of formulas describing the behavior of solutions for $k \geq m_{n}+$ 2 given in Theorems 4-9. In this part, we explain how this property can be used when the stability of a weakly delayed system (1) is considered. Since not all of the initial data have an impact on the behavior of the solution $x=x(k)$ as $k \geq m_{n}+$ 2 , a part of them can be fixed and, under such an assumption, we define a so-called conditional stability below. The fixing of some of the initial data leads to an unexpected phenomenon; 
the zero solution can be conditionally stable in spite of the fact that it is unstable in terms of the traditional definition of stability.

Define a norm of a $2 \times 2$ matrix $\mathscr{A}=\left(\mathscr{A}_{i j}\right), i, j=1,2$, as

$$
\|\mathscr{A}\|:=\max \left\{\left|\mathscr{A}_{11}\right|+\left|\mathscr{A}_{12}\right|,\left|\mathscr{A}_{21}\right|+\left|\mathscr{A}_{22}\right|\right\}
$$

and, for $2 \times 1$ vectors $x=\left(x_{1}, x_{2}\right)^{T}$, a vector norm

$$
\|x\|=\max \left\{\left|x_{1}\right|,\left|x_{2}\right|\right\} .
$$

For a discrete vector-function $\psi: Z_{-m_{n}}^{0} \rightarrow R^{2}$, we define

$$
\begin{aligned}
& \|\psi\|_{m_{n}} \\
& \quad:=\max \left\{\left\|\psi\left(-m_{n}\right)\right\|,\left\|\psi\left(-m_{n}+1\right)\right\|, \ldots,\|\psi(0)\|\right\} .
\end{aligned}
$$

In the following definition, the notion of conditional stability is explained. The two first parts of it are the classical definitions of stability and can be found, for example, in $[2,3]$.

Definition 10. The zero solution $x(k)=\theta, k \in Z_{-m_{n}}^{\infty}$, of (1) is said to be

(a) stable if, given $\varepsilon>0$ and $k_{0} \geq 0$, there exists $\delta=$ $\delta\left(\varepsilon, k_{0}\right)$ such that $\varphi(k), k \in Z_{k_{0}-m_{n}}^{k_{0}},\|\varphi\|_{m_{n}}<\delta$ implies $\left\|x\left(k, k_{0}, \varphi\right)\right\|<\varepsilon$ for all $k \geq k_{0}$, uniformly stable if $\delta$ may be chosen independently of $k_{0}$, and unstable if it is not stable;

(b) asymptotically stable if it is stable and $\lim _{k \rightarrow \infty}\|x(k)\|=0$;

(c) conditionally stable (conditionally asymptotically stable) if it is unstable (not asymptotically stable), but if it is stable (asymptotically stable) under the condition that there exists a fixed subspace $P \subset \mathbb{R}^{2\left(m_{n}+1\right)}, 1 \leq$ $\operatorname{dim} P<2\left(m_{n}+1\right)$, and the initial data satisfy

$$
\left\{\varphi\left(-m_{n}\right), \varphi\left(-m_{n}+1\right), \ldots, \varphi(0)\right\} \in \mathbb{R}^{2\left(m_{n}+1\right)} \backslash P .
$$

Utilizing the formulas on the representation of solutions of (1), we prove results on conditional stability (since the existence of the subspaces $P$ in the proofs is obvious, we do not write them explicitly).

Theorem 11. If the assumptions of Theorem 4 hold, $\left|\lambda_{1}\right| \leq q<$ $1,\left|\lambda_{2}\right| \geq 1$, and $\varphi_{2}^{*}(0)=0$, then the zero solution of (1) is conditionally asymptotically stable.

Proof. In this case, $\varphi^{*}(0)=\left(\varphi_{1}^{*}(0), \varphi_{2}^{*}(0)\right)^{T}=\left(\varphi_{1}^{*}(0), 0\right)^{T}$ and $\Phi_{2}(0)=\left(\varphi_{2}^{*}(0), 0\right)^{T}=\theta$. From formula $\left(\mathrm{I}_{4}\right)$ in Theorem 4 , for $k \in Z_{m_{n}+2}^{\infty}$, we get

$$
\begin{gathered}
\|y(k)\| \leq\left\|\Lambda_{1}^{k} \varphi^{*}(0)\right\|+\| \sum_{l=1}^{n} b_{12}^{* l}\left[\sum_{r=0}^{m_{l}} \lambda_{1}^{k-1-r} \Phi_{2}\left(r-m_{l}\right)\right. \\
\left.+\Phi_{2}(0) \sum_{r=m_{l}+1}^{k-1} \lambda_{1}^{k-1-r} \lambda_{2}^{r-m_{l}}\right] \| \\
\leq\left\|\left(\begin{array}{cc}
\lambda_{1}^{k} & 0 \\
0 & \lambda_{2}^{k}
\end{array}\right)\left(\begin{array}{c}
\varphi_{1}^{*}(0) \\
0
\end{array}\right)\right\|+\sum_{l=1}^{n}\left|b_{12}^{* l}\right|\left[\sum_{r=0}^{m_{l}}\left|\lambda_{1}\right|^{k-1-r}\right.
\end{gathered}
$$

$$
\begin{aligned}
& \left.\cdot\left\|\Phi_{2}\left(r-m_{l}\right)\right\|+\left\|\Phi_{2}(0)\right\| \sum_{r=m_{l}+1}^{k-1}\left|\lambda_{1}\right|^{k-1-r}\left|\lambda_{2}\right|^{r-m_{l}}\right] \\
& \leq\left|\lambda_{1}\right|^{k}\left\|\varphi_{1}^{*}(0)\right\|+\sum_{l=1}^{n}\left|b_{12}^{* l}\right|\left[\sum_{r=0}^{m_{l}}\left|\lambda_{1}\right|^{k-1-r}\right. \\
& \left.\cdot\left\|\Phi_{2}\left(r-m_{l}\right)\right\|\right] \leq q^{k}\left\|\varphi^{*}\right\|_{m_{n}}+\sum_{l=1}^{n}\left|b_{12}^{* l}\right|\left[\sum_{r=0}^{m_{l}} q^{k-1-r}\right. \\
& \left.\cdot\left\|\varphi^{*}\right\|_{m_{n}}\right] \leq\left[q^{k}+\sum_{l=1}^{n}\left|b_{12}^{* l}\right| \sum_{r=0}^{m_{l}} q^{k-1-r}\right]\left\|\varphi^{*}\right\|_{m_{n}} \\
& \leq\left[q^{k}+\sum_{l=1}^{n}\left|b_{12}^{* l}\right|\left(q^{k-1-m_{l}} \frac{1-q^{m_{l}+1}}{1-q}\right)\right]\left\|\varphi^{*}\right\|_{m_{n}} \\
& =q^{k}\left[1+\sum_{l=1}^{n}\left|b_{12}^{* l}\right| \frac{q^{-m_{l}-1}-1}{1-q}\right]\left\|\varphi^{*}\right\|_{m_{n}} .
\end{aligned}
$$

Now, it is easy to see that

$$
\begin{aligned}
& \lim _{k \rightarrow \infty}\|y(k)\| \\
& \quad \leq\left[1+\sum_{l=1}^{n}\left|b_{12}^{* l}\right| \frac{q^{-m_{l}-1}-1}{1-q}\right]\left\|\varphi^{*}\right\|_{m_{n}} \lim _{k \rightarrow \infty} q^{k}=0 ;
\end{aligned}
$$

that is, the zero solution is conditionally asymptotically stable. If $\varphi_{2}^{*}(0) \neq 0$, then from formula $\left(\mathrm{I}_{4}\right)$ in Theorem 4 , we get

$$
y_{2}(k)=\lambda_{2}^{k} \varphi_{2}^{*}(0) \neq 0
$$

as $k \geq m_{n}+2$, and the zero solution is unstable if $\left|\lambda_{2}\right|>1$ and not asymptotically stable if $\left|\lambda_{2}\right|=1$.

Similarly, with the aid of formula $\left(\mathrm{II}_{4}\right)$, Theorem 5 , the following theorem can be proved.

Theorem 12. If the assumptions of Theorem 5 hold, $\left|\lambda_{2}\right| \leq q<$ $1,\left|\lambda_{1}\right| \geq 1$, and $\varphi_{1}^{*}(0)=0$, then the zero solution of (1) is conditionally asymptotically stable.

Theorem 13. If the assumptions of Theorem 4 hold, $\left|\lambda_{1}\right|=1$, $\left|\lambda_{2}\right|>1$, and $\varphi_{2}^{*}(0)=0$, then the zero solution of (1) is conditionally stable.

Proof. We utilize formula $\left(\mathrm{I}_{4}\right)$ in Theorem 4 again. Since $\varphi^{*}(0)=\left(\varphi_{1}^{*}(0), \varphi_{2}^{*}(0)\right)^{T}=\left(\varphi_{1}^{*}(0), 0\right)^{T}$ and $\Phi_{2}(0)=$ $\left(\varphi_{2}^{*}(0), 0\right)^{T}=\theta$, for $k \in Z_{m_{n}+2}^{\infty}$, we get

$$
\begin{gathered}
\|y(k)\| \leq\left\|\Lambda_{1}^{k} \varphi^{*}(0)\right\|+\| \sum_{l=1}^{n} b_{12}^{* l}\left[\sum_{r=0}^{m_{l}} \lambda_{1}^{k-1-r} \Phi_{2}\left(r-m_{l}\right)\right. \\
\left.+\Phi_{2}(0) \sum_{r=m_{l}+1}^{k-1} \lambda_{1}^{k-1-r} \lambda_{2}^{r-m_{l}}\right] \| \\
\leq\left\|\left(\begin{array}{cc}
\lambda_{1}^{k} & 0 \\
0 & \lambda_{2}^{k}
\end{array}\right)\left(\begin{array}{c}
\varphi_{1}^{*}(0) \\
0
\end{array}\right)\right\|+\sum_{l=1}^{n}\left|b_{12}^{* l}\right|\left[\sum_{r=0}^{m_{l}}\left|\lambda_{1}\right|^{k-1-r}\right.
\end{gathered}
$$




$$
\begin{aligned}
& \cdot\left\|\Phi_{2}\left(r-m_{l}\right)\right\|+\left\|\Phi_{2}(0)\right\| \sum_{r=m_{l}+1}^{k-1}\left|\lambda_{1}\right|^{k-1-r} \\
& \left.\cdot\left|\lambda_{2}\right|^{r-m_{l}}\right] \leq\left|\lambda_{1}\right|^{k}\left\|\varphi_{1}^{*}(0)\right\|+\sum_{l=1}^{n}\left|b_{12}^{* l}\right|\left[\sum_{r=0}^{m_{l}}\left|\lambda_{1}\right|^{k-1-r}\right. \\
& \left.\cdot\left\|\Phi_{2}\left(r-m_{l}\right)\right\|\right] \leq\left\|\varphi^{*}\right\|_{m_{n}}+\sum_{l=1}^{n}\left|b_{12}^{* l}\right|\left[\sum_{r=0}^{m_{l}}\left\|\varphi^{*}\right\|_{m_{n}}\right] \\
& \leq\left[1+\sum_{l=1}^{n}\left|b_{12}^{* l}\right|\left(m_{l}+1\right)\right]\left\|\varphi^{*}\right\|_{m_{n}} .
\end{aligned}
$$

We set

$$
\begin{aligned}
M & :=1+\sum_{l=1}^{n}\left|b_{12}^{* l}\right|\left(m_{l}+1\right), \\
\delta & :=\frac{\varepsilon}{M}
\end{aligned}
$$

Then

$$
\|y(k)\| \leq M\left\|\varphi^{*}\right\|_{m_{n}}<\varepsilon, \quad k \in Z_{m_{n}+2}^{\infty}
$$

if $\left\|\varphi^{*}\right\|_{m_{n}}<\delta$. As in the proof of Theorem 11, we can show that if $\varphi_{2}^{*}(0) \neq 0$, the zero solution is unstable.

Theorem 14. If the assumptions of Theorem 5 hold, $\left|\lambda_{2}\right|=1$, $\left|\lambda_{1}\right|>1$, and $\varphi_{1}^{*}(0)=0$, then the zero solution of (1) is conditionally stable.

The proof can be performed similarly to that of Theorem 13 with the aid of formula $\left(\mathrm{II}_{4}\right)$, Theorem 5 .

Theorem 15. If the assumptions of Theorem 6 hold, $|\lambda|=1$ and $\varphi_{2}^{*}(0)=0$, then the zero solution of (1) is conditionally stable.

Proof. We have $\varphi^{*}(0)=\left(\varphi_{1}^{*}(0), \varphi_{2}^{*}(0)\right)^{T}=\left(\varphi_{1}^{*}(0), 0\right)^{T}$ and $\Phi_{2}(0)=\left(\varphi_{2}^{*}(0), 0\right)^{T}=\theta$. For $k \in Z_{m_{n}+2}^{\infty}$, we get by Theorem 6 , formula $\left(\mathrm{a}_{4}\right)$,

$$
\begin{gathered}
\|y(k)\| \leq\left\|\Lambda_{2}^{k} \varphi^{*}(0)\right\|+\| \sum_{l=1}^{n} b_{12}^{* l}\left[\sum_{r=0}^{m_{l}} \lambda^{k-1-r} \Phi_{2}\left(r-m_{l}\right)\right. \\
\left.+\left(k-1-m_{l}\right) \lambda^{k-1-m_{l}} \Phi_{2}(0)\right] \| \\
\leq\left\|\left(\begin{array}{cc}
\lambda^{k} & 0 \\
0 & \lambda^{k}
\end{array}\right)\left(\begin{array}{c}
\varphi_{1}^{*}(0) \\
0
\end{array}\right)\right\|+\sum_{l=1}^{n}\left|b_{12}^{* l}\right|\left[\sum_{r=0}^{m_{l}}|\lambda|^{k-1-r}\right. \\
\left.\cdot\left\|\Phi_{2}\left(r-m_{l}\right)\right\|+\left(k-1-m_{l}\right)|\lambda|^{k-1-m_{l}}\left\|\Phi_{2}(0)\right\|\right] \\
\leq\left\|\varphi_{1}^{*}(0)\right\|+\sum_{l=1}^{n}\left|b_{12}^{* l}\right|\left[\sum_{r=0}^{m_{l}}\left\|\varphi^{*}\right\|_{m_{n}}+\left(k-1-m_{l}\right)\right. \\
\left..\left\|\Phi_{2}(0)\right\|\right] \leq\left[1+\sum_{l=1}^{n}\left|b_{12}^{* l}\right|\left(m_{l}+1\right)\right]\left\|\varphi^{*}\right\|_{m_{n}} .
\end{gathered}
$$

Let $M$ and $\delta$ be given by (27). Then, the last equality implies

$$
\|y(k)\| \leq M\left\|\varphi^{*}\right\|_{m_{n}}<\varepsilon, \quad k \in Z_{m_{n}+2}^{\infty}
$$

if $\left\|\varphi^{*}\right\|_{m_{n}}<\delta$. So conditional stability is proved. If $\varphi_{2}^{*}(0) \neq 0$, then $\Phi_{2}(0)=\left(\varphi_{2}^{*}(0), 0\right)^{T} \neq \theta$ and formula $\left(\mathrm{a}_{4}\right)$ yields

$$
\begin{aligned}
& y_{1}(k)=\lambda^{k} \varphi_{1}^{*}(0)+\sum_{l=1}^{n} b_{12}^{* l}\left[\sum_{r=0}^{m_{l}} \lambda^{k-1-r} \varphi_{2}^{*}\left(r-m_{l}\right)\right. \\
& \left.\quad+\left(k-1-m_{l}\right) \lambda^{k-1-m_{l}} \varphi_{2}^{*}(0)\right] .
\end{aligned}
$$

Then, the zero solution is unstable since, obviously,

$$
\lim _{k \rightarrow \infty} \sup \left\|y_{1}(k)\right\|=\infty \text {. }
$$

The following two theorems can be proved using a scheme similar to that of the proof of Theorem 15 and with the aid of formula $\left(b_{4}\right)$, Theorem 7 , and formula $\left(c_{4}\right)$, Theorem 8 , respectively.

Theorem 16. If the assumptions of Theorem 7 hold, $|\lambda|=1$ and $\varphi_{1}^{*}(0)=0$, then the zero solution of (1) is conditionally stable.

Theorem 17. If the assumptions of Theorem 8 hold, $|\lambda|=1$ and $\varphi_{1}^{*}(0)=\varphi_{2}^{*}(0)=0$, then the zero solution of $(1)$ is conditionally stable.

Theorem 18. If the assumptions of Theorem 9 hold, $|\lambda|=1$ and $\varphi_{2}^{*}(0)=0$, then the zero solution of (1) is conditionally stable.

Proof. We show that the zero solution of (1) is conditionally stable. For $\left\|y_{1}(k)\right\|, k \in Z_{m_{n}+2}^{\infty}$, we get (by $4\left(\mathrm{y}_{1}\right)$, Theorem 9)

$$
\begin{aligned}
& \left\|y_{1}(k)\right\| \leq|\lambda|^{k}\left\|\varphi_{1}^{*}(0)\right\|+k|\lambda|^{k-1}\left\|\varphi_{2}^{*}(0)\right\|+\| \sum_{l=1}^{n} b_{12}^{* l} \\
& \cdot\left[\sum_{r=0}^{m_{l}} \lambda^{k-1-r} \varphi_{2}^{*}\left(r-m_{l}\right)\right. \\
& \left.+\left(k-1-m_{l}\right) \lambda^{k-1-m_{l}} \varphi_{2}^{*}(0)\right]\|\leq\| \varphi_{1}^{*}(0) \| \\
& +\sum_{l=1}^{n}\left|b_{12}^{* l}\right|\left[\sum_{r=0}^{m_{l}}\left\|\varphi^{*}\right\|_{m_{n}}\right] \leq\left[1+\sum_{l=1}^{n}\left|b_{12}^{* l}\right|\left(m_{l}+1\right)\right] \\
& \cdot\left\|\varphi^{*}\right\|_{m_{n}},
\end{aligned}
$$

and, for $\left\|y_{2}(k)\right\|, k \in Z_{m_{n}+2}^{\infty}$, we get (by $5\left(y_{2}\right)$, Theorem 9)

$$
\left\|y_{2}(k)\right\| \leq|\lambda|^{k}\left\|\varphi_{2}^{*}(0)\right\|=0, \quad k \in Z_{m_{n}+2}^{\infty}
$$

From (33), (34), it is easy to see that the zero solution of (1) is conditionally stable. If $\varphi_{2}^{*}(0) \neq 0$, then (by $5\left(y_{2}\right)$, Theorem 9)

$$
\limsup _{k \rightarrow \infty}\left\|y_{2}(k)\right\|=\infty
$$

and the zero solution is unstable. 


\section{Asymptotic Formulas}

Carefully analyzing the analytical formulas for the solutions of system (1) given in Theorems 4-9, it is possible (under various assumptions for the roots of (6) and for the initial data) to derive asymptotic formulas for the solutions of system (1) or simplify the formulas for the exact solutions. Below, we do such investigation. Set

$$
\begin{aligned}
& J_{1}:=\sum_{l=1}^{n} b_{12}^{* l}\left[\sum_{r=0}^{m_{l}} \lambda_{1}^{-r} \varphi_{2}^{*}\left(r-m_{l}\right)\right], \\
& J_{2}:=\sum_{l=1}^{n} b_{21}^{* l}\left[\sum_{r=0}^{m_{l}} \lambda_{2}^{-r} \varphi_{1}^{*}\left(r-m_{l}\right)\right] .
\end{aligned}
$$

The symbol $\sim$ used below stands for what is called the asymptotic equivalence. By definition, two nonzero real functions $z_{1}(k)$ and $z_{2}(k)$ defined for $k \rightarrow \infty$ are asymptotically equivalent if $\lim _{k \rightarrow \infty} z_{1}(k) / z_{2}(k)=1$.

Theorem 19. If the assumptions of Theorem 4 hold, then the solution of the initial problem (1), (3) is

$$
x(k)=S y(k)=S\left(y_{1}(k), y_{2}(k)\right)^{T}, \quad k \in \mathbb{Z}_{-m_{n}}^{\infty},
$$

where $y_{2}(k)=\lambda_{2}^{k} \varphi_{2}^{*}(0), k \in \mathbb{Z}_{1}^{\infty}$, and

(1) $y_{1}(k) \sim\left(\varphi_{1}^{*}(0)+J_{1} \lambda_{1}^{-1}-\varphi_{2}^{*}(0)\left(\lambda_{2} /\left(\lambda_{2}-\right.\right.\right.$ $\left.\left.\left.\lambda_{1}\right)\right) \sum_{l=1}^{n} b_{12}^{* l} \lambda_{1}^{-1-m_{l}}\right) \lambda_{1}^{k}$ if $k \rightarrow \infty,\left|\lambda_{1}\right|>\left|\lambda_{2}\right|$ and $\varphi_{1}^{*}(0)+J_{1} \lambda_{1}^{-1}-\varphi_{2}^{*}(0)\left(\lambda_{2} /\left(\lambda_{2}-\lambda_{1}\right)\right) \sum_{l=1}^{n} b_{12}^{* l} \lambda_{1}^{-1-m_{l}} \neq 0$,

(2) $y_{1}(k)=0$, if $k \in \mathbb{Z}_{m_{n}+2}^{\infty}$ and $\varphi_{1}^{*}(0)=J_{1}=\varphi_{2}^{*}(0)=0$,

(3) $y_{1}(k)=\varphi_{2}^{*}(0)\left(\lambda_{2} /\left(\lambda_{2}-\lambda_{1}\right)\right) \lambda_{2}^{k} \sum_{l=1}^{n} b_{12}^{* l} \lambda_{2}^{-1-m_{l}}$ if $k \epsilon$ $\mathbb{Z}_{m_{n}+2}^{\infty}, \lambda_{1} \neq 0$ and $\varphi_{1}^{*}(0)+J_{1} \lambda_{1}^{-1}-\varphi_{2}^{*}(0)\left(\lambda_{2} /\left(\lambda_{2}-\right.\right.$ $\left.\left.\lambda_{1}\right)\right) \sum_{l=1}^{n} b_{12}^{* l} \lambda_{1}^{-1-m_{l}}=0$,

(4) $y_{1}(k) \sim \varphi_{2}^{*}(0)\left(\lambda_{2} /\left(\lambda_{2}-\lambda_{1}\right)\right) \lambda_{2}^{k} \sum_{l=1}^{n} b_{12}^{* l} \lambda_{2}^{-1-m_{l}}$ if $k \rightarrow$ $\infty$ and $\left|\lambda_{1}\right|<\left|\lambda_{2}\right|$ and $\varphi_{2}^{*}(0) \sum_{l=1}^{n} b_{12}^{* l} \lambda_{2}^{-1-m_{l}} \neq 0$,

(5) $y_{1}(k)=\lambda_{1}^{k} \varphi_{1}^{*}(0)+J_{1} \lambda_{1}^{k-1}$ if $k \in \mathbb{Z}_{m_{n}+2}^{\infty}$ and $\varphi_{2}^{*}(0)=0$,

(6) $y_{1}(k)=\lambda_{1}^{k} \varphi_{1}^{*}(0)+J_{1} \lambda_{1}^{k-1}+\left(\varphi_{2}^{*}(0) /\right.$ 2) $\sum_{l=1}^{n} b_{12}^{* l}\left((-1)^{k-1-m_{l}}-1\right) \lambda_{1}^{k-1-m_{l}}$ if $k \in \mathbb{Z}_{m_{n}+2}^{\infty}$ and $\lambda_{2}=-\lambda_{1}$.

Proof. Let $\lambda_{1} \neq 0$. Then

$$
\sum_{r=m_{l}+1}^{k-1} \lambda_{1}^{k-1-r} \lambda_{2}^{r-m_{l}}=\frac{\lambda_{2}}{\lambda_{2}-\lambda_{1}}\left(\lambda_{2}^{k-1-m_{l}}-\lambda_{1}^{k-1-m_{l}}\right)
$$

and formula $\left(\mathrm{I}_{4}\right)$, Theorem 4 , for solution $y(k), k \in \mathbb{Z}_{m_{n}+2}^{\infty}$, can be written as

$$
\begin{aligned}
y(k)= & \Lambda_{1}^{k} \varphi^{*}(0)+\left(J_{1}, 0\right)^{T} \lambda_{1}^{k-1} \\
& +\Phi_{2}(0) \frac{\lambda_{2}}{\lambda_{2}-\lambda_{1}} \sum_{l=1}^{n} b_{12}^{* l}\left(\lambda_{2}^{k-1-m_{l}}-\lambda_{1}^{k-1-m_{l}}\right) .
\end{aligned}
$$

From (39), we get

$$
\begin{aligned}
y_{1}(k)= & \lambda_{1}^{k} \varphi^{*}(0)+J_{1} \lambda_{1}^{k-1} \\
& +\varphi_{2}^{*}(0) \frac{\lambda_{2}}{\lambda_{2}-\lambda_{1}} \sum_{l=1}^{n} b_{12}^{* l}\left(\lambda_{2}^{k-1-m_{l}}-\lambda_{1}^{k-1-m_{l}}\right) .
\end{aligned}
$$

Then (40), if $\left|\lambda_{1}\right|>\left|\lambda_{2}\right|$, implies the statement (1). The statement (2) is an obvious consequence of (40). For $\lambda_{1}=0$, we get the statement (2) immediately from $\left(\mathrm{I}_{4}\right)$. Statement (3) is also a straightforward consequence of (40). Let $\left|\lambda_{1}\right|<\left|\lambda_{2}\right|$. Then from (40) we deduce

$$
\begin{aligned}
y_{1}(k) & \sim \varphi_{2}^{*}(0) \frac{\lambda_{2}}{\lambda_{2}-\lambda_{1}} \sum_{l=1}^{n} b_{12}^{* l} \lambda_{2}^{k-1-m_{l}} \\
& =\left(\varphi_{2}^{*}(0) \frac{\lambda_{2}}{\lambda_{2}-\lambda_{1}} \sum_{l=1}^{n} b_{12}^{* l} \lambda_{2}^{-1-m_{l}}\right) \lambda_{2}^{k}
\end{aligned}
$$

if $\varphi_{2}^{*}(0)\left(\lambda_{2} /\left(\lambda_{2}-\lambda_{1}\right)\right) \sum_{l=1}^{n} b_{12}^{* l} \lambda_{2}^{-1-m_{l}} \neq 0$ and the statement (4) is valid. If $\lambda_{1}=0$, then $\left(\mathrm{I}_{4}\right)$ implies

$$
y_{1}(k)=\varphi_{2}^{*}(0) \frac{\lambda_{2}}{\lambda_{2}-\lambda_{1}} \sum_{l=1}^{n} b_{12}^{* l} \lambda_{2}^{k-1-m_{l}}
$$

and the statement (4) remains true. The statement (5) follows from (40) if $\lambda_{1} \neq 0$. From this representation, we derive the above asymptotic formulas if $\lambda_{1} \neq 0$. If $\lambda_{1}=0$, then, by $\left(\mathrm{I}_{4}\right), y_{1}(k)=0$ and the statement (5) remains valid. The last statement (6) is a consequence of (40) and remains valid also if $\lambda_{1}=0$.

Theorem 20. If the assumptions of Theorem 5 hold, then the solution of the initial problem (1), (3) is

$$
x(k)=S y(k)=S\left(y_{1}(k), y_{2}(k)\right)^{T}, \quad k \in \mathbb{Z}_{-m_{n}}^{\infty},
$$

where $y_{1}(k)=\lambda_{1}^{k} \varphi_{1}^{*}(0), k \in \mathbb{Z}_{1}^{\infty}$, and

(1) $y_{2}(k) \sim\left(\varphi_{2}^{*}(0)+J_{2} \lambda_{2}^{-1}-\varphi_{1}^{*}(0)\left(\lambda_{1} /\left(\lambda_{1}-\right.\right.\right.$ $\left.\left.\left.\lambda_{2}\right)\right) \sum_{l=1}^{n} b_{21}^{* l} \lambda_{2}^{-1-m_{l}}\right) \lambda_{2}^{k}$ if $k \rightarrow \infty,\left|\lambda_{2}\right|>\left|\lambda_{1}\right|$ and $\varphi_{2}^{*}(0)+J_{2} \lambda_{2}^{-1}-\varphi_{1}^{*}(0)\left(\lambda_{1} /\left(\lambda_{1}-\lambda_{2}\right)\right) \sum_{l=1}^{n} b_{21}^{* l} \lambda_{2}^{-1-m_{l}} \neq 0$,

(2) $y_{2}(k)=0$ if $k \in \mathbb{Z}_{m_{n}+2}$ and $\varphi_{2}^{*}(0)=J_{2}=\varphi_{1}^{*}(0)=0$,

(3) $y_{2}(k)=\varphi_{1}^{*}(0)\left(\lambda_{1} /\left(\lambda_{1}-\lambda_{2}\right)\right) \lambda_{1}^{k} \sum_{l=1}^{n} b_{21}^{* l} \lambda_{1}^{-1-m_{l}}$ if $k \epsilon$ $\mathbb{Z}_{m_{n}+2}^{\infty}, \lambda_{2} \neq 0$, and $\varphi_{2}^{*}(0)+J_{2} \lambda_{2}^{-1}-\varphi_{1}^{*}(0)\left(\lambda_{1} /\left(\lambda_{1}-\right.\right.$ $\left.\left.\lambda_{2}\right)\right) \sum_{l=1}^{n} b_{21}^{* l} \lambda_{2}^{-1-m_{l}}=0$,

(4) $y_{2}(k) \sim \varphi_{1}^{*}(0)\left(\lambda_{1} /\left(\lambda_{1}-\lambda_{2}\right)\right) \lambda_{1}^{k} \sum_{l=1}^{n} b_{21}^{* l} \lambda_{1}^{-1-m_{l}}$ if $k \rightarrow$ $\infty,\left|\lambda_{2}\right|<\left|\lambda_{1}\right|$, and $\varphi_{1}^{*}(0) \sum_{l=1}^{n} b_{21}^{* l} \lambda_{1}^{-1-m_{l}} \neq 0$,

(5) $y_{2}(k)=\lambda_{2}^{k} \varphi_{2}^{*}(0)+J_{2} \lambda_{2}^{k-1}$ if $k \in \mathbb{Z}_{m_{n}+2}^{\infty}$ and $\varphi_{1}^{*}(0)=0$,

(6) $y_{2}(k)=\lambda_{2}^{k} \varphi_{2}^{*}(0)+J_{2} \lambda_{2}^{k-1}+\left(\varphi_{1}^{*}(0) /\right.$ 2) $\sum_{l=1}^{n} b_{21}^{* l}\left((-1)^{k-1-m_{l}}-1\right) \lambda_{2}^{k-1-m_{l}}$ if $k \in \mathbb{Z}_{m_{n}+2}^{\infty}$ and $\lambda_{1}=-\lambda_{2}$. 
Proof. Let $\lambda_{2} \neq 0$. Then,

$$
\sum_{r=m_{l}+1}^{k-1} \lambda_{1}^{r-m_{l}} \lambda_{2}^{k-1-r}=\frac{\lambda_{1}}{\lambda_{1}-\lambda_{2}}\left(\lambda_{1}^{k-1-m_{l}}-\lambda_{2}^{k-1-m_{l}}\right),
$$

and formula $\left(\mathrm{II}_{4}\right)$ of Theorem 5 for the solution $y(k), k \in$ $\mathbb{Z}_{m_{n}+2}^{\infty}$, can be written as

$$
\begin{aligned}
y(k)= & \Lambda_{1}^{k} \varphi^{*}(0)+\left(0, J_{2}\right)^{T} \lambda_{2}^{k-1} \\
& +\Phi_{1}(0) \frac{\lambda_{1}}{\lambda_{1}-\lambda_{2}} \sum_{l=1}^{n} b_{21}^{* l}\left(\lambda_{1}^{k-1-m_{l}}-\lambda_{2}^{k-1-m_{l}}\right) .
\end{aligned}
$$

From (45), we get

$$
\begin{aligned}
y_{2}(k)= & \lambda_{2}^{k} \varphi_{2}^{*}(0)+J_{2} \lambda_{2}^{k-1} \\
& +\varphi_{1}^{*}(0) \frac{\lambda_{1}}{\lambda_{1}-\lambda_{2}} \sum_{l=1}^{n} b_{21}^{* l}\left(\lambda_{1}^{k-1-m_{l}}-\lambda_{2}^{k-1-m_{l}}\right) .
\end{aligned}
$$

Now, to prove statements (1)-(6) of the theorem, we can proceed in much the same way as in the proof of Theorem 19.

Theorem 21. If the assumptions of Theorem 6 hold, then the solution of the initial problem (1), (3) is

$$
x(k)=S y(k)=S\left(y_{1}(k), y_{2}(k)\right)^{T}, \quad k \in \mathbb{Z}_{-m_{n}}^{\infty},
$$

where $y_{2}(k)=\lambda^{k} \varphi_{2}^{*}(0), k \in \mathbb{Z}_{1}^{\infty}$, and

(1) $y_{1}(k) \sim\left(\varphi_{2}^{*}(0) \sum_{l=1}^{n} b_{12}^{* l}\left(k-1-m_{l}\right) \lambda^{-1-m_{l}}\right) \lambda^{k}$ ifk $\rightarrow \infty$, $\varphi_{2}^{*}(0) \neq 0$ and $\lambda \neq 0$,

(2) $y_{1}(k)=\lambda^{k} \varphi_{1}^{*}(0)+J_{1} \lambda^{k-1}$ if $k \in \mathbb{Z}_{m_{n}+2}^{\infty}$ and $\varphi_{2}^{*}(0)=0$.

Proof. Formula $\left(\mathrm{a}_{4}\right)$, Theorem 6, can be written as

$$
\begin{aligned}
y(k)= & \Lambda_{2}^{k} \varphi^{*}(0)+\left(J_{1}, 0\right)^{T} \lambda^{k-1} \\
& +\sum_{l=1}^{n} b_{12}^{* l}\left(k-1-m_{l}\right) \lambda^{k-1-m_{l}} \Phi_{2}(0), \\
y_{1}(k)= & \lambda^{k} \varphi_{1}^{*}(0)+J_{1} \lambda^{k-1} \\
& +\sum_{l=1}^{n} b_{12}^{* l}\left(k-1-m_{l}\right) \lambda^{k-1-m_{l}} \varphi_{2}^{*}(0) .
\end{aligned}
$$

If $\varphi_{2}^{*}(0) \neq 0$ and $\lambda \neq 0$, then the last addition term is the leading term and formula (1) is proved. If $\varphi_{2}^{*}(0)=0$, we get formula (2).

Theorem 22. If the assumptions of Theorem 7 hold, then the solution of the initial problem (1), (3) is

$$
x(k)=S y(k)=S\left(y_{1}(k), y_{2}(k)\right)^{T}, \quad k \in \mathbb{Z}_{-m_{n}}^{\infty},
$$

where $y_{1}(k)=\lambda^{k} \varphi_{1}^{*}(0), k \in \mathbb{Z}_{1}^{\infty}$, and

(1) $y_{2}(k) \sim\left(\varphi_{1}^{*}(0) \sum_{l=1}^{n} b_{21}^{* l}\left(k-1-m_{l}\right) \lambda^{-1-m_{l}}\right) \lambda^{k}$ if $k \rightarrow \infty$, $\varphi_{1}^{*}(0) \neq 0$, and $\lambda \neq 0$,

(2) $y_{2}(k)=\lambda^{k} \varphi_{2}^{*}(0)+J_{2} \lambda^{k-1}$ if $k \in \mathbb{Z}_{m_{n}+2}^{\infty}$ and $\varphi_{1}^{*}(0)=0$.
Proof. Formula $\left(\mathrm{b}_{4}\right)$, Theorem 7 , can be written as

$$
\begin{aligned}
y(k)= & \Lambda_{2}^{k} \varphi^{*}(0)+\left(0, J_{2}\right)^{T} \lambda^{k-1} \\
& +\sum_{l=1}^{n} b_{21}^{* l}\left(k-1-m_{l}\right) \lambda^{k-1-m_{l}} \Phi_{1}(0), \\
y_{2}(k)= & \lambda^{k} \varphi_{2}^{*}(0)+J_{2} \lambda^{k-1} \\
& +\sum_{l=1}^{n} b_{21}^{* l}\left(k-1-m_{l}\right) \lambda^{k-1-m_{l}} \varphi_{1}^{*}(0) .
\end{aligned}
$$

Further, we can proceed as in the proof of Theorem (34). (20)

The following theorem uses the vector norm, given by

Theorem 23. If the assumptions of Theorem 8 hold and $\lambda \neq 0$, then the solution of the initial problem (1), (3) is

$$
x(k)=S y(k)=S\left(y_{1}(k), y_{2}(k)\right)^{T}, \quad k \in \mathbb{Z}_{-m_{n}}^{\infty},
$$

where

(1) $y(k) \sim \lambda^{k-1} \sum_{l=1}^{n}\left(k-1-m_{l}\right) \lambda^{-m_{l}} \Phi_{l}^{*}(0)$ if $k \rightarrow \infty$, $\sum_{l=1}^{n}\left\|\Phi_{l}^{*}(0)\right\| \neq 0$,

(2) $y(k)=\lambda^{k} \varphi^{*}(0)+\lambda^{k-1} \sum_{l=1}^{n} \sum_{r=0}^{m_{l}-1} \lambda^{-r} \Phi_{l}^{*}\left(r-m_{l}\right)$, if $k \in \mathbb{Z}_{m_{n}+2}^{\infty}, \sum_{l=1}^{n}\left\|\Phi_{l}^{*}(0)\right\|=0$.

Proof. Formula $\left(\mathrm{c}_{4}\right)$, Theorem 8, can be written as

$$
\begin{aligned}
y(k)= & \Lambda_{2}^{k} \varphi^{*}(0)+\lambda^{k-1} \sum_{l=1}^{n} \sum_{r=0}^{m_{l}} \lambda^{-r} \Phi_{l}^{*}\left(r-m_{l}\right) \\
& +\sum_{l=1}^{n}\left(k-1-m_{l}\right) \lambda^{k-1-m_{l}} \Phi_{l}^{*}(0)
\end{aligned}
$$

if $k \in \mathbb{Z}_{m_{n}+2}^{\infty}$. If $\sum_{l=1}^{n}\left\|\Phi_{l}^{*}(0)\right\| \neq 0$, then the second sum is a leading term in this formula. If $\sum_{l=1}^{n}\left\|\Phi_{l}^{*}(0)\right\|=0$, the first two terms are leading.

Theorem 24. If the assumptions of Theorem 9 hold, then the solution of the initial problem (1), (3) is

$$
x(k)=S y(k)=S\left(y_{1}(k), y_{2}(k)\right)^{T}, \quad k \in \mathbb{Z}_{-m_{n}}^{\infty},
$$

where $y_{2}(k)=\lambda^{k} \varphi_{2}^{*}(0)$ if $k \in \mathbb{Z}_{1}^{\infty}$ and

$$
\begin{aligned}
& \text { (1) } y_{1}(k) \sim k \lambda^{k-1} \varphi_{2}^{*}(0)+\sum_{l=1}^{n} b_{12}^{* l}\left(k-1-m_{l}\right) \lambda^{k-1-m_{l}} \varphi_{2}^{*}(0) \\
& \text { if } k \rightarrow \infty, \varphi_{2}^{*}(0) \neq 0 \text { and } \lambda \neq 0, \\
& \text { (2) } y_{1}(k)=\lambda^{k} \varphi_{1}^{*}(0)+J_{1} \lambda^{k-1} \text { if } k \in \mathbb{Z}_{m_{n}+2}^{\infty} \text { and } \varphi_{2}^{*}(0)=0 .
\end{aligned}
$$

Proof. The formula $4\left(\mathrm{y}_{1}\right)$, Theorem 9 , can be written as

$$
\begin{aligned}
y_{1}(k)= & \lambda^{k} \varphi_{1}^{*}(0)+k \lambda^{k-1} \varphi_{2}^{*}(0)+J_{1} \lambda^{k-1} \\
& +\sum_{l=1}^{n} b_{12}^{* l}\left(k-1-m_{l}\right) \lambda^{k-1-m_{l}} \varphi_{2}^{*}(0)
\end{aligned}
$$

if $k \in \mathbb{Z}_{m_{n}+2}^{\infty}$. The second and fourth terms are leading terms if $\varphi_{2}^{*}(0) \neq 0$ and $\lambda \neq 0$. If $\varphi_{2}^{*}(0)=0$, then the first and third terms are leading terms. 


\section{Example}

To illustrate the results derived, consider the following example. The computations below were partly implemented by WolframAlpha software (http://www.wolframalpha.com/). Let system (1) be reduced to

$$
\begin{aligned}
x_{1}(k+1)= & -6.5 x_{1}(k)+3.5 x_{2}(k)+4 x_{1}(k-1) \\
& -2 x_{2}(k-1)-2 x_{1}(k-2) \\
& +x_{2}(k-2), \\
x_{2}(k+1)= & -7 x_{1}(k)+4 x_{2}(k)+8 x_{1}(k-1) \\
& -4 x_{2}(k-1)-4 x_{1}(k-2) \\
& +2 x_{2}(k-2)
\end{aligned}
$$

and let initial problem (3) be

$$
x(k)=\varphi(k)=(1,1)^{T}, \quad k \in \mathbb{Z}_{-2}^{0} .
$$

In this case, $n=2, m_{1}=1, m_{2}=2$,

$$
\begin{aligned}
A & =\left(\begin{array}{cc}
-6.5 & 3.5 \\
-7 & 4
\end{array}\right), \\
B_{1} & =\left(\begin{array}{cc}
4 & -2 \\
8 & -4
\end{array}\right), \\
B_{2} & =\left(\begin{array}{ll}
-2 & 1 \\
-4 & 2
\end{array}\right) .
\end{aligned}
$$

The eigenvalues of $A$ are $\lambda_{1}=0.5, \lambda_{2}=-3$. System (55) is weakly delayed by Theorem 3 . Indeed, (8) holds since

$$
\begin{aligned}
& b_{11}^{1}+b_{22}^{1}=4-4=0, \\
& b_{11}^{2}+b_{22}^{2}=-2+2=0
\end{aligned}
$$

(9) holds since

$$
\begin{aligned}
& \left|\begin{array}{ll}
b_{11}^{1} & b_{12}^{1} \\
b_{21}^{1} & b_{22}^{1}
\end{array}\right|=\left|\begin{array}{rr}
4 & -2 \\
8 & -4
\end{array}\right|=0, \\
& \left|\begin{array}{ll}
b_{11}^{2} & b_{12}^{2} \\
b_{21}^{2} & b_{22}^{2}
\end{array}\right|=\left|\begin{array}{ll}
-2 & 1 \\
-4 & 2
\end{array}\right|=0,
\end{aligned}
$$

(10) holds since

$$
\begin{aligned}
& \left|\begin{array}{ll}
a_{11} & a_{12} \\
b_{21}^{1} & b_{22}^{1}
\end{array}\right|+\left|\begin{array}{ll}
b_{11}^{1} & b_{12}^{1} \\
a_{21} & a_{22}
\end{array}\right|=\left|\begin{array}{cc}
-6.5 & 3.5 \\
8 & -4
\end{array}\right|+\left|\begin{array}{cc}
4 & -2 \\
-7 & 4
\end{array}\right|=0, \\
& \left|\begin{array}{ll}
a_{11} & a_{12} \\
\mathrm{~b}_{21}^{2} & b_{22}^{2}
\end{array}\right|+\left|\begin{array}{ll}
b_{11}^{2} & b_{12}^{2} \\
a_{21} & a_{22}
\end{array}\right|=\left|\begin{array}{cc}
-6.5 & 3.5 \\
-4 & 2
\end{array}\right|+\left|\begin{array}{ll}
-2 & 1 \\
-7 & 4
\end{array}\right|=0,
\end{aligned}
$$

and (11) holds since

$$
\left|\begin{array}{ll}
b_{11}^{1} & b_{12}^{1} \\
b_{21}^{2} & b_{22}^{2}
\end{array}\right|+\left|\begin{array}{ll}
b_{11}^{2} & b_{12}^{2} \\
b_{21}^{1} & b_{22}^{1}
\end{array}\right|=\left|\begin{array}{cc}
4 & -2 \\
-4 & 2
\end{array}\right|+\left|\begin{array}{cc}
-2 & 1 \\
8 & -4
\end{array}\right|=0 .
$$

A nonsingular matrix $S$ transforming $A$ to the corresponding Jordan form is

$$
\begin{aligned}
S & =\left(\begin{array}{ll}
1 & 1 \\
2 & 1
\end{array}\right), \\
S^{-1} & =\left(\begin{array}{cc}
-1 & 1 \\
2 & -1
\end{array}\right) .
\end{aligned}
$$

Then,

$$
\begin{aligned}
\Lambda_{1} & =S^{-1} A S=\left(\begin{array}{cc}
-1 & 1 \\
2 & -1
\end{array}\right)\left(\begin{array}{cc}
-6.5 & 3.5 \\
-7 & 4
\end{array}\right)\left(\begin{array}{ll}
1 & 1 \\
2 & 1
\end{array}\right) \\
& =\left(\begin{array}{cc}
0.5 & 0 \\
0 & -3
\end{array}\right), \\
\Lambda_{1}^{k} & =\left(\begin{array}{cc}
(0.5)^{k} & 0 \\
0 & (-3)^{k}
\end{array}\right), \quad k \in \mathbb{Z}_{0}^{\infty}, \\
B_{1}^{*} & =S^{-1} B_{1} S=\left(\begin{array}{cc}
-1 & 1 \\
2 & -1
\end{array}\right)\left(\begin{array}{cc}
4 & -2 \\
8 & -4
\end{array}\right)\left(\begin{array}{ll}
1 & 1 \\
2 & 1
\end{array}\right) \\
& =\left(\begin{array}{cc}
0 & 2 \\
0 & 0
\end{array}\right), \\
B_{2}^{*} & =S^{-1} B_{2} S=\left(\begin{array}{cc}
-1 & 1 \\
2 & -1
\end{array}\right)\left(\begin{array}{ll}
-2 & 1 \\
-4 & 2
\end{array}\right)\left(\begin{array}{ll}
1 & 1 \\
2 & 1
\end{array}\right) \\
& =\left(\begin{array}{cc}
0 & -1 \\
0 & 0
\end{array}\right) .
\end{aligned}
$$

The transformed initial problem (56) is

$$
\varphi^{*}(k)=S^{-1} \varphi(k)=S^{-1}(1,1)^{T}=(0,1)^{T}, \quad k \in \mathbb{Z}_{-2}^{0} .
$$

The transformed system (55) with the initial problem is

$$
\begin{array}{r}
y(k+1)=\Lambda_{1} y(k)+B_{1}^{*} y(k-1)+B_{2}^{*} y(k-2), \\
k \in \mathbb{Z}_{0}^{\infty},
\end{array}
$$

where

$$
y(k)=\varphi^{*}(k)=\left(\varphi_{1}^{*}(k), \varphi_{2}^{*}(k)\right)=(0,1)^{T},
$$

or

$$
\begin{aligned}
& y_{1}(k+1)=0.5 y_{1}(k)+2 y_{2}(k-1)-x_{2}(k-2), \\
& y_{2}(k+1)=-3 y_{2}(k),
\end{aligned}
$$

where the initial data are

$$
\begin{aligned}
& y_{1}(k)=0, \\
& y_{2}(k)=1,
\end{aligned}
$$

$$
k \in \mathbb{Z}_{-2}^{0}
$$


The function $\Phi_{2}: \mathbb{Z}_{-2}^{0} \rightarrow \mathbb{R}^{2}$ is defined as

$$
\Phi_{2}(k):=\left(\varphi_{2}^{*}(k), 0\right)^{T}=(1,0)^{T}, \quad k \in \mathbb{Z}_{-2}^{0} .
$$

All assumptions of Theorem 4 are true. Therefore, the solution of the initial problem (55), (56) is $x(k)=S y(k)$, $k \in \mathbb{Z}_{-2}^{\infty}$, where

$$
\begin{aligned}
& \left(\mathrm{I}_{1}^{*}\right) y(k)=\left(y_{1}(k), y_{2}(k)\right)=\left(\varphi_{1}^{*}(k), \varphi_{2}^{*}(k)\right)=(0,1)^{T} \text { if } k \epsilon \\
& \mathbb{Z}_{-2}^{0} \\
& \left(\mathrm{I}_{2}^{*}\right) y(k)=\Lambda_{1}^{k} \varphi^{*}(0)+\sum_{r=0}^{k-1} \lambda_{1}^{k-1-r} \sum_{l=1}^{2} b_{12}^{* l} \Phi_{2}\left(r-m_{l}\right)= \\
& \left(\begin{array}{cc}
(0.5)^{k} & 0 \\
0 & (-3)^{k}
\end{array}\right)\left(\begin{array}{l}
0 \\
1
\end{array}\right)+\left(\begin{array}{l}
1 \\
0
\end{array}\right) \sum_{r=0}^{k-1}(0.5)^{k-1-r} \text { if } k \in \mathbb{Z}_{1}^{2}, \\
& \left(\mathrm{I}_{3}^{*}\right) y(3)=\Lambda_{1}^{3} \varphi^{*}(0)+\sum_{r=0}^{2} \lambda_{1}^{2-r} b_{12}^{* 2} \Phi_{2}(r-2) \\
& +b_{12}^{* 1}\left[\sum_{r=0}^{1} \lambda_{1}^{2-r} \Phi_{2}(r \quad-\quad 1) \quad+\right. \\
& \left.\Phi_{2}(0) \lambda_{2}\right]=\left(\begin{array}{cc}
(0.5)^{3} & 0 \\
0 & (-3)^{3}
\end{array}\right)\left(\begin{array}{l}
0 \\
1
\end{array}\right)-\left(\begin{array}{l}
1 \\
0
\end{array}\right) \sum_{r=0}^{2}(0.5)^{2-r}+ \\
& \left(\begin{array}{l}
2 \\
0
\end{array}\right)\left[\sum_{r=0}^{1}(0.5)^{2-r}-3\right] \\
& \left(\mathrm{I}_{4}^{*}\right) y(k)=\Lambda_{1}^{k} \varphi^{*}(0)+\sum_{l=1}^{2} b_{12}^{* l}\left[\sum_{r=0}^{m_{l}} \lambda_{1}^{k-1-r} \Phi_{2}\left(r-m_{l}\right)\right. \\
& \left.+\Phi_{2}(0) \sum_{r=m_{l}+1}^{k-1} \lambda_{1}^{k-1-r} \lambda_{2}^{r-m_{l}}\right]=\left(\begin{array}{cc}
(0.5)^{k} & 0 \\
0 & (-3)^{k}
\end{array}\right)\left(\begin{array}{l}
0 \\
1
\end{array}\right)+ \\
& \left(\begin{array}{l}
2 \\
0
\end{array}\right)\left[\sum_{r=0}^{1}(0.5)^{k-1-r}+\sum_{r=2}^{k-1}(0.5)^{k-1-r}(-3)^{r-1}\right]+ \\
& \left(\begin{array}{c}
-1 \\
0
\end{array}\right)\left[\sum_{r=0}^{2}(0.5)^{k-1-r}+\sum_{r=3}^{k-1}(0.5)^{k-1-r}(-3)^{r-2}\right] \quad \text { if } \\
& k \in \mathbb{Z}_{4}^{\infty} \text {. }
\end{aligned}
$$

The asymptotic behavior of the solutions of systems satisfying the assumptions of Theorem 4 is covered by Theorem 19 . In the considered case, the assumptions of formula (4) in Theorem 19 are satisfied. Then,

$$
\begin{aligned}
y_{2}(k) & =\lambda_{2}^{k} \varphi_{2}^{*}(0)=(-3)^{k}, \quad k \in \mathbb{Z}_{1}^{\infty}, \\
y_{1}(k) & \sim \varphi_{2}^{*}(0) \frac{\lambda_{2}}{\lambda_{2}-\lambda_{1}} \lambda_{2}^{k} \sum_{l=1}^{n} b_{12}^{* l} \lambda_{2}^{-1-m_{l}} \\
& =\frac{-3}{-3-0.5}(-3)^{k}\left(2(-3)^{-2}-1(-3)^{-3}\right) \\
& =2(-3)^{k-2} \quad \text { if } k \longrightarrow \infty ;
\end{aligned}
$$

that is,

$$
y_{1}(k) \sim 2(-3)^{k-2} \quad k \longrightarrow \infty .
$$

The last formula can be deduced from $\left(\mathrm{I}_{4}^{*}\right)$ as it is done (in the general case) in the proof of Theorem 19.

Finally, we select initial data for system (55) to derive a result on conditional asymptotic stability by Theorem 11 . Let, for $k=0$, the initial data be given by the equation

$$
x(0)=\varphi(0)=\left(\varphi_{1}(0), \varphi_{2}(0)\right)^{T}=(c, 2 c)^{T},
$$

where $c$ is an arbitrary constant. Then, the initial data $y(0)$ for (65), deduced from (16), are

$$
\begin{aligned}
y(0) & =\varphi^{*}(0)=\left(\varphi_{1}^{*}(0), \varphi_{2}^{*}(0)\right)^{T}=S^{-1} \varphi(0) \\
& =S^{-1}(c, 2 c)^{T}=(c, 0)^{T} .
\end{aligned}
$$

Since $\left|\lambda_{1}\right|=0.5<1,|\lambda|_{2}=3>1$, and $\varphi_{2}^{*}(0)=0$, all assumptions of Theorem 11 are true. Therefore, the zero solution of system (55) is conditionally stable if the initial data

$$
x(k)=\varphi(k)=\left(\varphi_{1}(k), \varphi_{2}(k)\right)^{T}, \quad k \in \mathbb{Z}_{-2}^{0}
$$

satisfy the equation $\left(\varphi_{1}(0), \varphi_{2}(0)\right)=(c, 2 c)$.

In the proof of Theorem 11 this statement is derived from formula $\left(\mathrm{I}_{4}\right)$ in Theorem 4 . In the considered particular case we have

$$
\begin{aligned}
\Phi_{2}(k) & :=\left(\varphi_{2}^{*}(k), 0\right)^{T}, \quad k \in \mathbb{Z}_{-2}^{-1}, \\
\Phi_{2}(0) & :=\left(\varphi_{2}^{*}(0), 0\right)^{T}=(0,0)^{T}, \\
y(k) & \\
= & \Lambda_{1}^{k}\left(\begin{array}{l}
c \\
0
\end{array}\right)+2(0,5)^{k-1}\left(\begin{array}{c}
\varphi_{2}^{*}(-1) \\
0
\end{array}\right) \\
= & \left.\left(\begin{array}{c}
c(0,5)^{k-1}\left(\begin{array}{c}
\varphi_{2}^{*}(-2) \\
0
\end{array}\right)+(0,5)^{k-2}\left(\varphi_{2}^{*}(-1)\right. \\
0
\end{array}\right)\right) \\
0 & (0,5)^{k-1}\left(\begin{array}{c}
\varphi_{2}^{*}(-2) \\
0
\end{array}\right) \\
= & \left(\begin{array}{c}
\left(c-2 \varphi_{2}^{*}(-2)\right)(0.5)^{k} \\
0
\end{array}\right) .
\end{aligned}
$$

Then

$$
\lim _{k \rightarrow \infty} y(k)=\theta
$$

since, by the above formula,

$$
\begin{aligned}
\lim _{k \rightarrow \infty} y_{1}(k) & =0, \\
y_{2}(k) & =0, \quad k \in \mathbb{Z}_{0}^{\infty} .
\end{aligned}
$$

\section{Conclusions}

The paper deals with conditional stability and asymptotic representation of solutions to linear weakly delayed discrete systems (1) if the Jordan form of the matrix A equals (12), (13), or (14), that is, $\Lambda_{1}, \Lambda_{2}$, or $\Lambda_{3}$. For these cases, conditional stability is investigated and formulas are deduced expressing the asymptotic behavior of solutions as $k \rightarrow \infty$. For further results related to weakly delayed systems, representations of solutions of discrete systems, their stability, and asymptotic behavior, we refer to [1-11] and to the references therein. It is an open question whether similar results can be derived in the case of system (1) consisting of three equations.

\section{Conflicts of Interest}

The authors declare that there are no conflicts of interest regarding the publication of this paper. 


\section{Acknowledgments}

The first author has been supported by the Project no. LO1408, AdMaS UP-Advanced Materials, Structures and Technologies (supported by Ministry of Education, Youth and Sports of the Czech Republic under the National Sustainability Programme I). The third author has been supported by the Grant FEKT-S-17-4225 of Faculty of Electrical Engineering and Communication, BUT.

\section{References}

[1] J. Diblík and H. Halfarová, "General explicit solution of planar weakly delayed linear discrete systems and pasting its solutions," Abstract and Applied Analysis, vol. 2014, Article ID 627295, 37 pages, 2014.

[2] R. P. Agarwal, Difference Equations and Inequalities: Theory, Methods, and Applications, CRC Press, Boca Raton, Fla, USA, 2000.

[3] S. N. Elaydi, An Introduction to Difference Equations, Springer, New York, NY, USA, 3rd edition, 2005.

[4] L. Berezansky and S. Pinelas, "Oscillation properties for a scalar linear difference equation of mixed type," Mathematica Bohemica, vol. 141, no. 2, pp. 169-182, 2016.

[5] L. Berezansky, M. Migda, and E. Schmeidel, "Some stability conditions for scalar Volterra difference equations," Opuscula Mathematica, vol. 36, no. 4, pp. 459-470, 2016.

[6] J. Diblík and H. Halfarová, "Explicit general solution of planar linear discrete systems with constant coefficients and weak delays," Advances in Difference Equations, vol. 2013, article 50, pp. 1-29, 2013.

[7] J. Diblík and D. Y. Khusainov, "Representation of solutions of discrete delayed system $x(k+1)=A x(k)+B x(k-m)+f(k)$ with commutative matrices," Journal of Mathematical Analysis and Applications, vol. 318, no. 1, pp. 63-76, 2006.

[8] J. Diblík, D. Y. Khusainov, and Z. Šmarda, "Construction of the general solution of planar linear discrete systems with constant coefficients and weak delay," Advances in Difference Equations, vol. 2009, Article ID 784935, 18 pages, 2009.

[9] J. Diblík and B. Morávková, "Representation of the solutions of linear discrete systems with constant coefficients and two delays," Abstract and Applied Analysis, vol. 2014, Article ID 320476, 19 pages, 2014.

[10] M. Migda, M. Růžičková, and E. Schmeidel, "Boundedness and stability of discrete Volterra equations," Advances in Difference Equations, vol. 2015, article 47, pp. 1-11, 2015.

[11] J. Morchało and M. Migda, "Boundedness of solutions of difference systems with delays," Computers \& Mathematics with Applications, vol. 64, no. 7, pp. 2233-2240, 2012. 


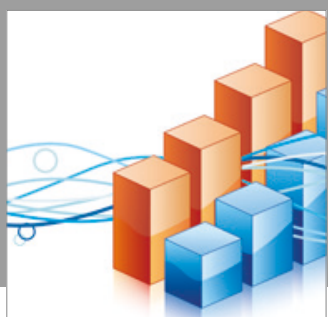

Advances in

Operations Research

vatersals

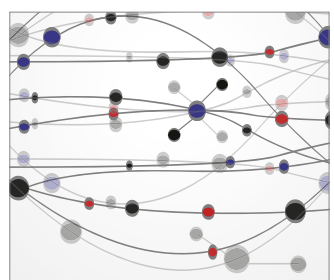

\section{The Scientific} World Journal
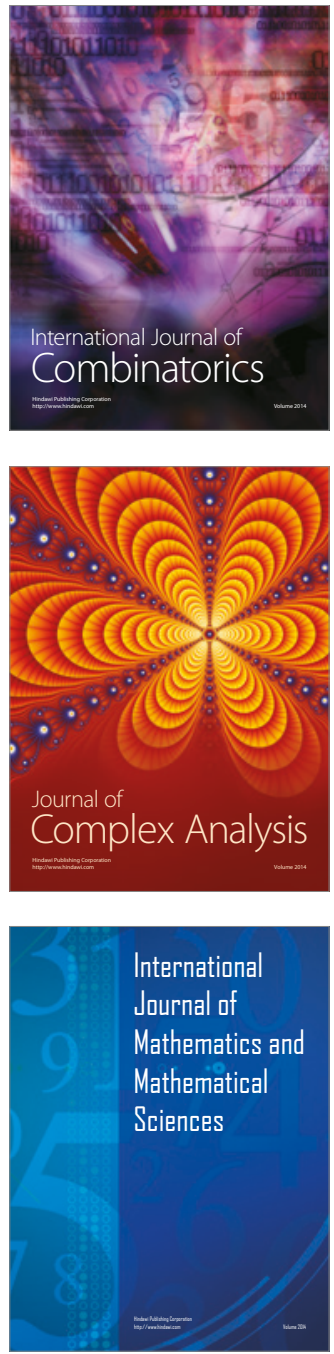
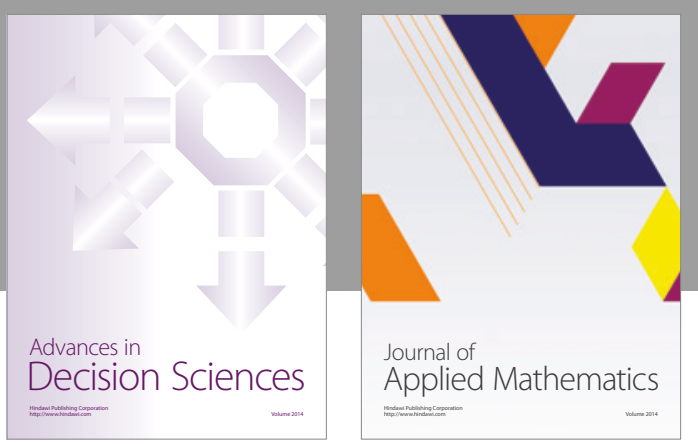

Algebra

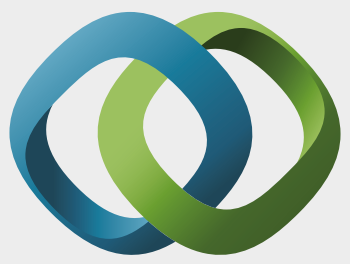

\section{Hindawi}

Submit your manuscripts at

https://www.hindawi.com
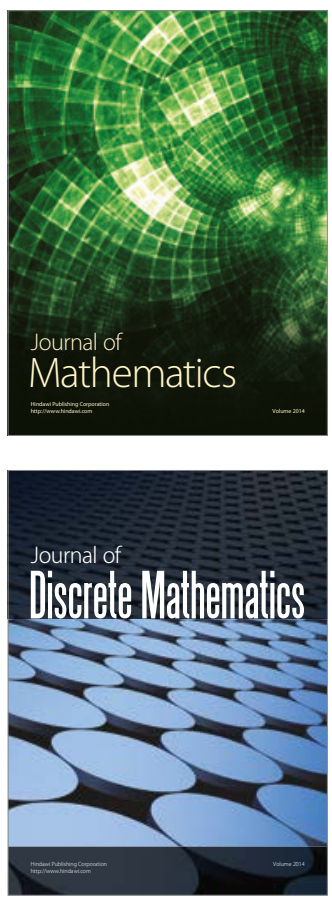

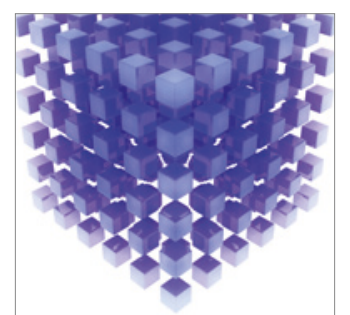

Mathematical Problems in Engineering
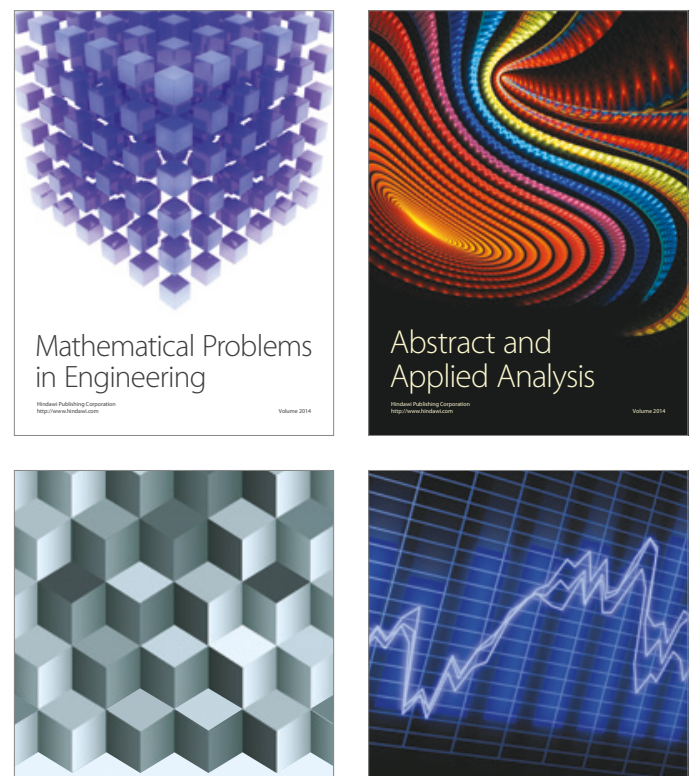

Journal of

Function Spaces

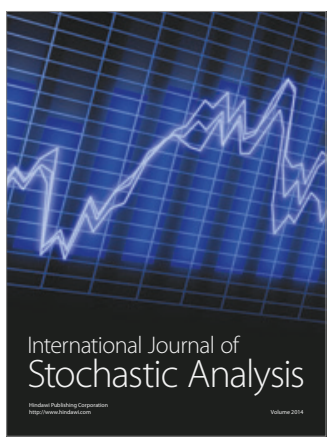

Probability and Statistics
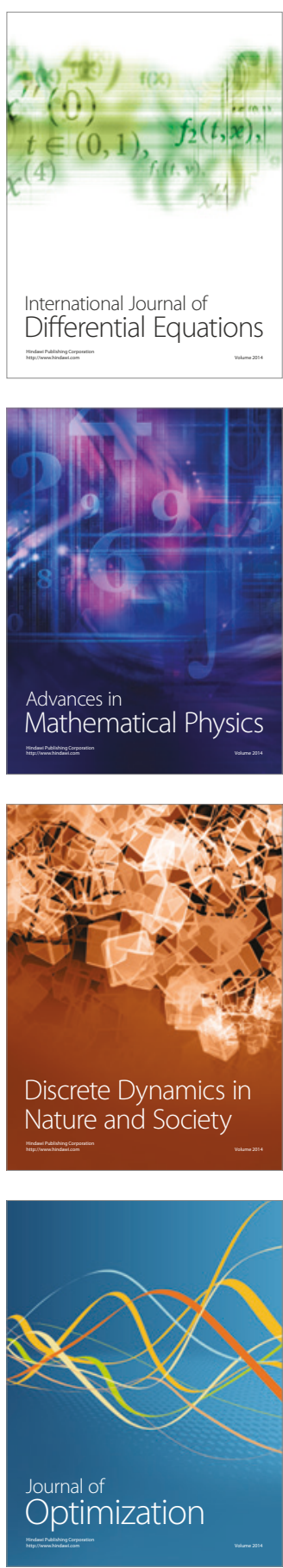